\title{
L'intégration des Antillais en France et aux Etats- Unis : contextes socio-institutionnels et processus de territorialisation
}

\section{Cédric Audebert}

\author{
(2) OpenEdition \\ Journals \\ Édition électronique \\ URL : https://journals.openedition.org/remi/4269 \\ DOI : $10.4000 /$ remi.4269 \\ ISSN : $1777-5418$ \\ Éditeur \\ Université de Poitiers \\ Édition imprimée \\ Date de publication : 1 avril 2008 \\ Pagination : 65-87 \\ ISBN : 978-2-917-627-48-2 \\ ISSN : 0765-0752
}

Référence électronique

Cédric Audebert, "L'intégration des Antillais en France et aux Etats-Unis : contextes socio-

institutionnels et processus de territorialisation », Revue européenne des migrations internationales [En ligne], vol. 24 - $n^{\circ} 1$ | 2008, mis en ligne le 01 avril 2011, consulté le 15 avril 2022. URL : http:// journals.openedition.org/remi/4269; DOI : https://doi.org/10.4000/remi.4269

Ce document a été généré automatiquement le 15 avril 2022.

(c) Université de Poitiers 


\title{
L'intégration des Antillais en France et aux Etats-Unis : contextes socio- institutionnels et processus de territorialisation
}

\author{
Cédric Audebert
}

1 Les migrations contemporaines vers les sociétés postindustrielles, et la question du «vivre ensemble» qui y est attachée tendent à renouveler la problématique de l'appartenance citoyenne (Constant, 2000; Grosfoguel, 1999). Si les deux formes traditionnelles que recouvre cette dernière - celles relatives aux droits civils et politiques - ont pu fournir un cadre politico-juridique favorable aux migrations antillaises ${ }^{1}$ vers leur métropole (Constant, 1987), sa récente dimension revendicative liée à l'accès aux droits sociaux met en lumière la «minorisation » de ces populations dans l'émigration et celle de leurs enfants (Giraud, 2002; Giraud, Marie, 1987)2. Leur implantation durable pose notamment la question de leur intégration dans la conception dominante de la "communauté nationale» et celle de leur accession légitime mais problématique à l'égalité sociale et à la reconnaissance identitaire.

2 Le choix de présenter l'expérience de trois populations issues de la matrice caribéenne - les Portoricains, les Antillais français, les Haïtiens - n'est pas neutre. Il permet de confronter les expériences des deux premières, qui ont la citoyenneté du pays d'installation, à celle de la troisième, qui est étrangère. Aux États-Unis, la présence en grand nombre des Portoricains date des années 1940 et celle des Haïtiens du début des années 1960. En France, les originaires des Antilles françaises et d'Haïti sont des dizaines de milliers dès les années 1960 pour les premiers et à partir de la fin des années 1980 pour les seconds ${ }^{3}$. Le choix de ces trois populations interroge aussi de manière comparative les effets des cadres socio-institutionnels des sociétés d'installation française et états-unienne sur les processus de territorialisation des populations migrantes. 
3 En complémentarité avec les travaux antérieurs de sciences politiques et de sociologie sur la citoyenneté et l'insertion sociale des originaires des Antilles, notre perspective géographique se propose de mettre en exergue la relation au territoire comme clé de compréhension des multiples expériences caribéennes dans des contextes variés. La France et les États-Unis présentent deux cadres socio-institutionnels spécifiques de gestion de la différence culturelle. L'approche de cette question par la territorialisation des migrants dans la ville constitue un apport du géographe à la compréhension de leur destin et du fonctionnement des sociétés d'accueil. On peut notamment s'interroger sur le rôle des processus de territorialisation des Antillais dans l'évolution des RELATIONS interethniques ${ }^{4}$ et la construction du fait communautaire. À partir d'une interrogation sur les enjeux sociaux de ces processus, on peut se demander dans quelle mesure le rapport à l'espace révèle des conceptions spécifiques du « vivre ensemble » en France et aux États-Unis.

4 Le rapport à l'espace et la dimension fonctionnelle du territoire ne se déclinent pas de la même manière de part et d'autre de l'Atlantique. L'expérience contemporaine des populations dans ces sociétés d'accueil révèle l'importance du contexte socioinstitutionnel, en particulier dans les politiques d'insertion de ces populations et dans le traitement institutionnel de la différence culturelle. Une approche en termes de géographie sociale et politique permettra de saisir le territoire à la fois comme un acteur et un enjeu du processus d'insertion et de construction de l'altérité, montrant simultanément les similitudes et les différences des deux sociétés dans leur rapport à l'espace.

\section{Construction de l'altérité et insertion sociale des Antillais en France et aux États-Unis}

5 En dépit d'évolutions divergentes, les constructions historiques de ces deux pays d'immigration ancienne comportent des similitudes : projets utopiques universalistes comparables, genèse de systèmes profondément inégalitaires (esclavage, systèmes internes de statuts différenciés sur une base ethnique), conception restrictive de la "communauté nationale» expliquant la résurgence périodique de préoccupations nativistes (Lacorne, 1997). Plus récemment, dans les années 1960 et 1970, les revendications régionales et mouvements de minorités contestant le pouvoir central ont révélé dans les deux pays une crise d'identité de l'État-nation (Green, 2002). Ces legs de l'histoire ont marqué de manière similaire les conceptions contemporaines $\mathrm{du}$ "vivre ensemble». Une différence majeure entre les cadres socio-institutionnels d'intégration français et états-unien aujourd'hui réside néanmoins dans le traitement institutionnel de la différence culturelle et la place accordée aux communautés migrantes ou au contraire la négation de leur existence, notamment dans la sphère politique.

6 Aux États-Unis, tandis que la communauté ethnicisée fournit un cadre culturel familier propice à une adaptation en douceur des nouveaux venus, la catégorie ethnique constitue le cadre institutionnel de leur intégration. Elle leur facilite l'accession aux ressources locales et fédérales - éducation, emploi et logement notamment lorsqu'ils sont considérés officiellement comme membres de minorités «ethniques». La jouissance de la citoyenneté et l'adhésion aux valeurs de la société d'accueil 
permettent aux membres de la communauté de s'organiser et de s'impliquer collectivement dans la politique locale pour défendre leurs intérêts.

7 L'appartenance communautaire devient alors un facteur d'intégration, à la différence de la République française universaliste qui appréhende la spécificité identitaire exprimée publiquement comme une atteinte à l'intégrité nationale. En France, l'indifférenciation formelle dans la sphère publique garantirait théoriquement un traitement équitable à tous les citoyens, condition de la résorption à terme des inégalités sociales. Cette conception horizontale de l'extension des droits de la citoyenneté accordés indépendamment des différences ne reconnaît dans l'espace public que l'individu. Les représentations de la citoyenneté et le champ plus ou moins important accordé au fait communautaire et ethnique ont des incidences sur le traitement des migrants antillais et la conception de leur légitimité à faire partie de la « communauté imaginée » (Anderson, 1983) dans l'Hexagone et outre-Atlantique.

\section{L'incorporation ambivalente des Antillais dans la communauté imaginée aux États-Unis et en France}

8 Aux États-Unis, la reconnaissance officielle de la réalité communautaire et de l'ethnicisation des rapports sociaux issue de la législation sur les droits civiques s'est traduite par la mise en place d'une catégorisation de la population en cinq grands groupes sur la base de l'origine géographique (Asians et Pacific Islanders), des caractéristiques culturelles (Hispanics) ou d'attributs phénotypiques (Blacks, Whites, Amerindians).

9 L'intégration des Haïtiens aux États-Unis est longtemps restée problématique du fait de deux obstacles. Le refus des autorités de reconnaître officiellement leur présence les a maintenus dans la clandestinité, et donc dans l'impossibilité de participer à la vie de la cité. Contrairement à l'idée répandue d'un groupe haïtien peu investi dans la politique du pays d'accueil (Basch, et al., 1994; Cohen, 2002), ce n'est pas la forte activité transnationale des Haïtiens qui a freiné leur participation à la vie politique étatsunienne, mais les conditions restrictives de cette participation qui les ont cantonnés dans une activité tournée vers le pays d'origine. Leur qualité de non-citoyens a ainsi lourdement pesé sur leurs perspectives d'intégration jusqu'aux années 1990.

10 Le deuxième obstacle à leur reconnaissance en tant que communauté a résidé dans leur catégorisation en tant que Blacks or African Americans. Cela a eu pour corollaire le passage au second plan de leur spécificité culturelle et de leurs priorités politiques visà-vis d'Haïti. Mais cela les a surtout placés dans une situation de double minorité les maintenant à la marge de la « communauté imaginée » états-unienne : minoritaires en tant que Noirs dans une société hiérarchisée sur une base ethno-raciale, ils l'ont aussi été en tant qu'immigrés créolophones au sein d'une population noire majoritairement anglophone (Audebert, 2006a). Nous verrons plus loin qu'ils ont répondu à cette situation par une mobilisation politique croissante, en particulier à partir des années 1990 lorsqu'ils ont pu devenir citoyens du pays d'accueil.

11 Indépendamment de leur qualité de citoyens états-uniens et parce qu'ils sont hispanophones d'origine, les Portoricains sont incorporés dans la catégorie des Hispaniques au même titre que les Cubains ou les Mexicains. Or, catégorisée sur la base de ses attributs linguistiques, la population hispanique est construite et représentée comme une minorité culturelle distincte de la majorité anglo-saxonne. L'immigration 
hispanique est perçue comme le principal moteur des mutations démographiques et culturelles de la société états-unienne contemporaine. La perception d'un "péril hispanique » dans une partie de l'opinion publique s'appuie sur des considérations tant démographiques (poids numérique de l'immigration mexicaine) et culturelles (expansion de la langue espagnole et de la religion catholique) que politiques (lobby cubain de Miami).

ésence hispanique constitue le principal objet des préoccupations néo-nativistes de Californie, du Texas et de Floride. La construction externe de l'altérité liée aux représentations de la majorité anglo-saxonne et à l'institution d'une catégorie hispanique semble l'emporter sur les différences culturelles internes à cette catégorie, ce qui contribue à créer le sentiment d'une communauté de destin et d'intérêts chez les Hispaniques aux États-Unis. La culture portoricaine a en particulier été l'objet de stéréotypes négatifs - relayés jusque dans la littérature et le cinéma - et reste perçue comme incompatible avec les valeurs anglo-saxonnes dominantes de la société étatsunienne (Grosfoguel, 2002).

Il apparaît donc que la qualité de citoyens états-uniens ne prémunit pas les Portoricains d'une relégation hors de la communauté nationale imaginée, au même titre que les immigrés haïtiens qui ne possèdent pas la citoyenneté du pays d'installation. Les incidences de la catégorisation ethno-raciale états-unienne apparaissent ambiguës. Créée dans la mouvance des droits civiques, celle-ci confère une visibilité culturelle à la communauté ethnicisée en lui fournissant le cadre institutionnel de son intégration. Mais elle contribue en même temps à produire, figer et essentialiser une altérité qui explique en partie la relégation et la discrimination. Le contexte républicain français permet-il de mieux répondre au défi de l'appartenance à la communauté nationale de ses migrants antillais?

14 L'idéologie républicaine basée sur l'égalité théorique de tous les citoyens introduit une distinction fondamentale en termes d'accession aux droits politiques et sociaux entre les migrants des DFA (Départements français d'Amérique) qui sont citoyens français et les immigrés haïtiens dont $63 \%$ ne possèdent pas la citoyenneté du pays d'accueil (INSEE, 1999). Une analyse plus approfondie de la question de la citoyenneté chez les originaires des Antilles françaises met néanmoins en exergue les limites d'un universalisme inachevé. Ce dernier créé en effet paradoxalement de l'altérité et de l'inégalité, par le discrédit d'identités ne correspondant pas à l'idéologie dominante de l'«identité nationale ». À l'instar des multiples héritages culturels du Maghreb ou de l'Afrique noire, l'expression de spécificités culturelles antillaises dans l'espace public dans l'Hexagone est perçue comme une atteinte à l'unité nationale davantage que comme une richesse héritée de la diversité culturelle de l'ex-empire colonial français.

15 Le paradoxe du modèle français réside dans une conception de l'intégration où les nouveaux apports migratoires ne transformeraient qu'à la marge l'héritage historique national. Cette idéologie s'inscrit en décalage avec la notion même d'intégration, fondée sur une dynamique d'interaction, de participation et sur les changements sociétaux qui en émanent. Quoique constitutifs de la construction nationale française, les événements historiques majeurs que sont la traite négrière et l'esclavage sont restés largement tabous jusqu'à très récemment, comme l'ont illustré les manuels scolaires d'histoire. Encore marqué par l'ignorance et l'indifférence, le rapport que la société française entretient avec ces événements est révélé par le caractère confidentiel de leur commémoration. En dépit de l'inscription du 10 mai comme date commémorative 
nationale de l'abolition de l'esclavage, ils restent perçus comme relevant spécifiquement des histoires antillo-guyanaise et réunionnaise, et non comme des événements constitutifs d'une histoire nationale appartenant à chaque citoyen. L'occultation des syncrétismes et productions culturelles nés de ces événements et qui se déploient au sein même de la République témoigne d'une crispation niant la réalité pluriculturelle française et rendant illégitime l'expression de l'altérité. La "minorisation" identitaire revêt un sens particulier lorsqu'elle est synonyme de relégation voire d'exclusion sociale ou vécue comme telle par les groupes concernés.

\section{L'insertion socio-économique des Antillais dans les espaces métropolitains : les effets de la « minorisation »} et Haïtiens - liée à deux évolutions. La première est le départ des classes ouvrières et moyennes des ghettos et «enclaves» les plus paupérisés; la seconde réside dans les mutations structurelles de l'économie urbaine ayant engendré la disparition des emplois manuels bien payés et le développement d'emplois de services peu rémunérés pour des travailleurs ayant un faible niveau de formation (Wilson, 1987). Au cours des années 1990, la croissance de l'emploi dans les inner cities paupérisées, de 8,5\%, a été deux fois plus faible que celle enregistrée dans les suburbs; et à la fin de la décennie, le revenu médian des ménages dans les inner cities - 33150 - n'équivalait qu'à $71 \%$ de celui des ménages des suburbs (U.S. Department of Housing and Urban Development, 2000). La désolidarisation sociale et spatiale s'est accompagnée d'un processus de "décitoyenneté ", soit une impossibilité de participer pleinement à la vie de la cité (Massey, Denton,1993).

En s'incarnant dans les rapports sociaux, la catégorisation ethno-raciale et l'assignation communautaire ont contribué dans une certaine mesure à une inégalité d'accès aux ressources et aux emplois. Implicitement classés au bas de l'échelle socio-économique états-unienne, les Haïtiens et Portoricains portent le poids de stéréotypes négatifs qui pèsent sur leur insertion sur le marché de l'emploi de la société d'accueil. Tandis que les Portoricains « portent les stigmates de la paresse, de la propension à la violence, parfois de la stupidité ou de la saleté » (Grosfoguel, 2002), les Haïtiens de Miami ont longtemps subi l'image de boat people sales, incultes, pauvres et hors-la-loi (Audebert, 2006a). On leur a même officiellement attribué à tort le lourd fardeau de la responsabilité de la propagation de l'épidémie de SIDA aux États-Unis (Stepick, 1998).

En France, la remise en cause des rapports sociaux hérités de la période industrielle, la dualisation du marché du travail et la difficulté des institutions de la République 
- l'école notamment - à traduire dans les faits les principes fondateurs d'égalité et de solidarité au bénéfice de tous incarnent la crise du modèle français. Or les minorités de facto - celles dont l'acceptation dans la communauté des citoyens est la plus problématique - apparaissent concernées au premier chef par cette crise de citoyenneté sur fond de reclassement socio-économique. Le nivellement du marché du travail par le haut dans un contexte de compétition internationale s'est accompagné d'une disqualification des travailleurs peu qualifiés, mais aussi de ceux issus des minorités dites « visibles".

La réalité sociale quotidienne d'une partie de la population issue des migrations antillaises en France comme aux États-Unis s'avère problématique et questionne l'idéal universaliste et égalitaire de ces deux sociétés aux mécanismes socio-institutionnels d'intégration si différents. Outre-Atlantique, les originaires d'Haïti et de Porto Rico comptent parmi les populations les plus défavorisées socialement. Ainsi, dans le comté de Miami-Dade qui rassemble la principale concentration d'Haïtiens des États-Unis, la part des Haïtiens occupant des emplois à bas salaire (31\%) est trois fois plus élevée que celle des Blancs non hispaniques (9\%). Le revenu médian de leur ménage de 27300 \$ n'équivaut qu'à à peine la moitié de celui des seconds, de 50000 \$ (U.S. Census Bureau, 2002). En conséquence, le taux de pauvreté des Haïtiens (30\%) est pratiquement le double de celui de la population en général, de $18 \%$ (idem).

21 Étant donné le caractère tardif de l'accession des Haïtiens de Floride à la citoyenneté états-unienne et de manière plus générale à l'obtention de papiers, il est tentant de mettre en avant les facteurs politico-juridiques pour expliquer leur relégation socioéconomique. Pourtant les Portoricains, citoyens états-uniens, ne semblent pas mieux lotis à New York, une ville où leur présence date de plus d'un demi-siècle. Leur taux de chômage y reste trois fois supérieur à celui des Blancs non hispaniques et leur taux de pauvreté près de quatre fois plus élevé - $33 \%$ contre $9 \%$ (U.S. Census Bureau, 1999). S'il a pu favoriser l'émergence d'une classe moyenne noire, le traitement institutionnel de la différence culturelle outre-Atlantique n'a pu qu'imparfaitement répondre aux attentes des minorités les plus discriminées et il apparaît légitime de se demander si la catégorisation ethno-raciale n'a pas des effets pervers.

La situation des originaires de la Caraïbe dans la société française pose également la question de l'accession de tous les citoyens aux droits sociaux. Aucun indicateur statistique de l'INSEE ne permet de répondre à cette question de manière aussi révélatrice que celui du chômage des jeunes originaires des départements d'outre-mer nés en métropole cité par Claude-Valentin Marie. Leur taux de chômage de 27,2\% - comparable à celui des originaires d'Haïti - témoignait d'une situation nettement plus défavorable que celle déjà peu enviable des jeunes Métropolitains ( $16 \%$ de chômeurs) en 1999 (Marie, 2002). Dans un contexte général de privatisation et de contraction de l'emploi public, les jeunes d'origine antillaise ont moins l'opportunité d'accéder à la fonction publique que l'avaient leurs parents, et sont davantage confrontés aux difficultés de l'insertion sur le marché de l'emploi. Dans le secteur privé, ils partagent une expérience commune de la discrimination avec leurs compatriotes d'origine subsaharienne ou maghrébine ${ }^{6}$.

Les expériences de ces trois populations caribéennes suggèrent que la jouissance de la citoyenneté ne constitue pas un rempart contre la marginalisation et qu'à l'échelle macro-sociale, la mise en pratique des idéaux républicains ne va pas de soi quand il s'agit de populations ne correspondant pas aux représentations dominantes de la 
" communauté nationale ». L'accession dans les faits aux droits sociaux attenants à la jouissance de la citoyenneté restant problématique, celle-ci apparaît de plus en plus questionnée (sans être pour autant désavouée) par d'autres référents identificatoires tels que la construction communautaire sur une base culturelle, celle de l'origine géographique ou celle du lieu de résidence. Il apparaît alors fécond de s'interroger sur l'éventuelle existence d'une relation réciproque entre constructions communautaires et processus de territorialisation dans les aires métropolitaines concernées.

\section{Les Antillais dans la ville en France et aux États-Unis : des modalités dissemblables de territorialisation}

L'incorporation problématique des Haïtiens, des Portoricains et des Antillais français et de leurs enfants dans la communauté nationale du point de vue des formes politiques et sociales de réalisation de la citoyenneté moderne a formalisé le cadre externe de la construction de l'altérité de ces populations. Celle-ci a progressivement été internalisée par des Caribéens déjà conscients de leur héritage culturel spécifique et réalisant dans les espaces de migration - à travers leur destin ou celui de leurs enfants l'importance de l'écart entre l'image idéalisée de la (néo-)métropole et les réalités mitigées de l'insertion sur le marché de l'emploi et plus généralement de l'intégration dans les communautés nationales. Il convient de s'interroger sur les incidences des deux «modèles » d'intégration sur la formalisation du rapport à l'altérité de ces migrants. Cette interrogation peut être menée à partir de leur apprentissage des territoires d'implantation dans les deux pays. Comment ces gestions distinctes de la différence culturelle se traduisent-elle dans les rapports à l'espace?

\section{L'espace résidentiel des Caribéens en France et aux États-Unis : des concentrations comparables marquant des logiques sociétales distinctes}

Les populations antillaises sont caractérisées par une concentration spatiale marquée, ayant pu favoriser leur structuration communautaire en France et aux États-Unis. Parmi tous les groupes de migrants dans les deux pays, les originaires des Antilles sont ceux qui enregistrent les taux de métropolisation et de concentration résidentielle les plus élevés. Ainsi, en France, plus de $70 \%$ des Antillais français et $87 \%$ des Haïtiens résident dans l'agglomération parisienne, contre $58 \%$ des immigrés d'Afrique francophone, 50 \% de ceux des pays d'Indochine (Viêt-Nam, Laos, Cambodge), 40 \% des immigrés portugais, $36 \%$ des immigrés algériens par exemple (INSEE, 1999). Aux ÉtatsUnis, $67 \%$ des Haïtiens résident dans les aires métropolitaines de New York et Miami ; et, en dépit d'une dispersion remarquable depuis les années 1970, $62 \%$ des Portoricains vivent encore dans la Mégalopolis et en particulier $39 \%$ à New York (U.S. Census Bureau, 2002). La concentration résidentielle des Antillais est aussi sensible à l'échelle intra-urbaine, le tiers des Portoricains de New York City habitant dans le Bronx et $38 \%$ des Haïtiens de Miami-Dade vivant dans le secteur de North Miami/Golden Glades (idem). d'autre de l'Atlantique offre une première grille de lecture du rapport différencié à 
l'altérité dans les deux sociétés. Certes, un certain nombre de caractéristiques communes à ces migrants permettent de rendre compte de leur concentration dans quelques grandes villes. Ces dernières, qui sont aussi les principaux pôles de l'activité économique et culturelle des pays d'installation, jouissent d'une image attractive auprès des migrants potentiels. Pour ces derniers, New York, Miami ou Paris symbolisent à elles seules les opportunités offertes par les sociétés d'accueil. En outre, la recherche d'un logement à moindre coût à proximité des zones d'emploi peu qualifié liée au statut social modeste d'une grande partie des Antillais rend compte de cette concentration, notamment dans des logements vétustes et exigus dans un premier temps. Enfin, les réseaux sociaux transatlantiques orientent les migrants vers les quartiers où sont déjà installés leurs compatriotes dans le cadre de migrations familiales renforçant la métropolisation.

Mais le cadre général précédemment analysé de l'incorporation ambivalente des Antillais dans les communautés nationales et de ses effets sur leur insertion sociale et économique s'incarne différemment dans leur rapport à l'espace et rend compte de logiques résidentielles distinctes selon le pays d'accueil. En France, la gestion institutionnelle d'une partie de la migration antillaise via le BUMIDOM $^{7}$ (Constant, 1987) et son insertion consécutive notable dans le fonctionnariat ont eu des incidences plus ou moins directes sur sa concentration spatiale. En effet, l'accession de près de la moitié des ménages antillais au logement social dans certaines banlieues et la mobilité résidentielle ultérieure relativement aisée dans le parc social réservé à l'administration (PTT, hôpitaux, assistance publique, éducation nationale) expliquent leur forte présence dans les zones est, nord et sud-est de l'agglomération parisienne (Condon, 1994). Le développement d'une pratique plus répandue de demande en logement social chez les Antillais de France les distingue nettement des pratiques de ceux des ÉtatsUnis dont les pratiques résidentielles consistent entre autres à éviter par tous les moyens les parcs de logement public (projects) qui renvoient une image socialement négative.

L'expérience d'une partie des Antillais dans l'Hexagone met donc en exergue des conditions spécifiques d'inscription sur le marché du logement. Si celles-ci sont en partie liées à la particularité de leur migration et des modalités de leur insertion et quoique certains bailleurs leur confèrent des caractéristiques spécifiques proches de la stigmatisation (surpopulation, nuisances sonores), ces conditions ne semblent pas forcément pour autant marquées par des mécanismes de mise à distance et de discrimination à grande échelle. Il en va différemment aux États-Unis, où leur expérience résidentielle porte l'empreinte d'une altérité marquant les territoires urbains.

Les pratiques des agents immobiliers, des ménages et des organismes fédéraux se traduisent notamment par une segmentation ethno-raciale des marchés du logement. Les Caribéens peuvent tout d'abord faire l'objet de stratégies discriminatoires de la part des agents immobiliers qui tendent à limiter l'entrée des Noirs dans les quartiers anglos et orientent la demande des ménages noirs vers des quartiers noirs. En outre, les préjugés des ménages blancs limitent leur installation dans des quartiers accueillant des Noirs en grand nombre et motivent leur départ progressif de quartiers où résident 20 à $25 \%$ de Noirs (Massey, Denton, 1993). Enfin, l'allocation de prêts immobiliers fédéraux privilégie les quartiers "homogènes" sur le plan social (couches sociales aisées) et ethnique (quartiers blancs) dont la valeur immobilière est perçue comme 
stable (idem). ${ }^{8}$ Parallèlement, la délimitation officielle de territoires antillais reconnus institutionnellement par les autorités locales sur la base de la présence de communautés ethnicisées - Little Haiti et Little Havana à Miami, South Bronx pour les Portoricains à New York par exemple - a renforcé le processus de concentration spatiale et illustre la manière dont l'ethnicisation des populations migrantes s'incarne dans le territoire aux États-Unis.

\section{Les territoires urbains, supports de la structuration communautaire antillaise : l'exemple des églises}

30 Quoique évolutive dans le temps, la géographie résidentielle des Caribéens a constitué un élément favorable à la genèse de fonctionnements communautaires, dont les églises et les commerces ethniques sont les points de repères ${ }^{9}$. Certains quartiers ou villes ont ainsi servi de cadre spatial à l'émergence d'une vie communautaire se déclinant différemment dans les deux pays. En France, la région parisienne et en particulier la Seine-Saint-Denis et le nord-est parisien s'affirment comme des épicentres de la vie communautaire des populations antillaises, où la religion joue un rôle fondamental. La multiplication des églises à forte orientation communautaire est liée à la recherche d'un cadre social familier fournissant repères culturels et affectifs dans l'émigration, à l'instar notamment des églises adventistes (Desplan, 2005).

31 Ces structures institutionnelles fortes permettent la réappropriation d'une identité antillaise (éventuellement réaménagée) par le développement d'actions sociales et culturelles et des modes d'expression liturgique en phase avec celles des sociétés d'origine mais aussi celles développées dans les banlieues. De ce point de vue, les prêches vivants et chants en créole sur fond de musique haïtienne ou antillo-guyanaise, de reggae ou de rap constituent un fait nouveau dans l'Hexagone, de même que la cohabitation plus ou moins importante au sein d'églises pentecôtistes et évangéliques de fidèles franco-antillais, haïtiens et africains. Cette dynamique religieuse n'est pas sans rappeler celle des communautés haïtiennes de Miami et New York. On y retrouve le même phénomène de concentration spatiale de la vie religieuse dans les quartiers populaires, où les hangars désaffectés et anciens locaux industriels sont réhabilités pour en faire des églises communautaires répondant aux besoins sociaux, culturels et psychologiques des immigrés.

Mais la comparaison avec les États-Unis s'arrête là. En effet, sur le territoire de la République, la présence de ces églises qualifiées d'« ethniques" ou d'« exotiques " - rassemblant a minima 10000 Haïtiens et 30000 Antillo-Guyanais rien qu'en Région parisienne - reste mal acceptée. Sur plusieurs centaines de communautés protestantes antillaises et/ou africaines en France, seules une trentaine sont reconnues officiellement dont une sur trois est implantée en Seine-Saint-Denis. Outre-Atlantique, le rôle institutionnel dévolu aux communautés ethniques et le faible cadre social d'intégration confèrent aux églises une reconnaissance officielle et un rôle social de premier plan, qui en France reste le domaine réservé de l'État. Le gouvernement fédéral délègue aux églises portoricaines du South Bronx et haïtiennes de Miami et de Brooklyn la fonction de centres de services sociaux en vue notamment de l'adaptation des migrants, montrant l'importance de la communauté et le rôle structurant du territoire ethnique dans le cadre socio-institutionnel états-unien. 

$\mathrm{du}$ lien social et de l'entraide et, à travers la reproduction transnationale des communautés de parenté, d'amitié et de voisinage, font de ces territoires des centralités polarisant les mobilités intra-urbaines et structurant les réseaux de sociabilité des immigrés. Le quartier de Little Haiti rassemble à peine plus de $15 \%$ de la population haïtienne de Miami, mais concentre $60 \%$ des églises et des commerces ethniques haïtiens de l'agglomération (Audebert, 2002, 2006a). Les institutions religieuses y sont des lieux de rencontre, de circulation de l'information et de mise en scène de la culture et de l'identité communautaires. Les quartiers populaires antillais aux États-Unis sont ainsi quadrillés par un maillage dense d'églises constituant l'une des principales marques de l'investissement de l'espace par les immigrés et sur lequel ils fondent leur identité. Si la stratégie des « paroisses nationales » s'est traduite par la création d'églises puissantes dominant l'ensemble de l'organisation catholique haïtienne et hispano-caribéenne de Floride (Corpus Christi, Notre Dame d'Haïti), celle des réseaux protestants transnationaux a abouti à l'implantation de multiples congrégations de taille variable et relativement autonomes. Little Haiti compte actuellement une église communautaire pour moins de 400 résidents d'origine haïtienne, un record outre-Atlantique !

\section{Construction de l'altérité et représentations spatiales}

Au-delà des points de repères que constituent les églises, mais aussi les commerces et les associations de migrants, la construction mentale de l'altérité s'opère à travers la représentation des territoires dans la ville. Les structures sociales «se convertissent progressivement en structures mentales et en systèmes de préférences » et "par le processus de l'apprentissage social, par les pratiques et les représentations du monde, chacun apprend donc à classer, en utilisant les typologies sociales en usage " (Bourdieu, 1993). Or une distinction remarquable entre les lieux de vie caribéens en France et aux États-Unis réside dans la multiethnicité plus affirmée des premiers par rapport aux seconds. Dans les banlieues françaises, la construction des représentations de soi et des « autres » s'élabore dans la cohabitation et l'échange à l'école ou dans la cité. L'identité de quartier, l'une des plus mises en avant par les jeunes d'origine antillaise nés en Ile-de-France, ne se confond pas rigoureusement avec celle de l'ethnicité puisque les quartiers des banlieues françaises sont fondamentalement des lieux de brassage et d'interaction entre des populations diverses partageant généralement dans les mêmes espaces de socialisation (MJC, milieu associatif, écoles, halls d'immeubles) des expériences communes de construction de soi. Les Antillais ne sont numériquement majoritaires dans aucun de leurs territoires résidentiels, qui sont aussi dans une large mesure ceux de populations d'origine subsaharienne, maghrébine, juive et européenne autochtone ou immigrée ${ }^{10}$.

Si les représentations collectives des migrants antillais vis-à-vis d'autres groupes portent l'empreinte de l'héritage colonial - relation au Métropolitain marquée par le rejet de rapports sociaux perçus comme hérités de la colonisation, distanciation vis-àvis de l'Africain et de l'Haïtien renvoyant une image dévalorisante construite dans un contexte colonial -, le positionnement de leurs enfants nés dans l'Hexagone apparaît plus complexe. En effet, ces derniers partagent avec les originaires d'Afrique subsaharienne l'expérience de la discrimination à l'emploi et parfois d'un racisme qu'illustrent les rapports avec la police et avec certains voisins «babtou ${ }^{11}$ de condition modeste et aux positions politiques radicales. En réponse aux idées préconçues de la

Revue européenne des migrations internationales, vol. 24 - $n^{\circ} 1$ | 2008 
société globale formalisant un groupe sur la seule base de la couleur de la peau, une partie des jeunes d'origine caribéenne met en scène une identité antillaise de France recoupant partiellement celle d'une identité "renoi» ou "black». Le glissement sémantique opéré sur une trentaine d'années des termes « Noir » et « Renoi » à celui de «Black» pour désigner les populations noires est symptomatique de l'évolution du regard que la société française porte sur elle-même et sur ses diverses composantes.

Le terme «black» calqué sur les Noirs de France puise sa source dans un contexte de mondialisation culturelle et médiatique puissamment impulsée par le monde anglosaxon, où sont amalgamés originaires des Antilles et d'Afrique dans l'Hexagone, et plus globalement Noirs français et anglo-saxons. Certains reprennent à leur compte cette labellisation car elle les valorise par une identification aux figures emblématiques noires états-uniennes et caribéennes du sport ou de la musique - en particulier la musique revendicative populaire (rap, dance hall) en résonance avec leurs problèmes quotidiens et indissociable d'une expression artistique multiforme éminemment urbaine sur laquelle ils fondent en partie leur estime de soi. Mais superficielle par l'effet de mode qui la motive et uniformisante par la négation de l'héritage culturel et de la complexité identitaire de populations d'origines diverses, cette appellation n'en demeure pas moins problématique. Elle s'avère notamment porteuse d'une nouvelle altérité en confortant les représentations de la population noire comme un groupe à part dans la société française ${ }^{12}$ et traduit l'émergence d'identités fantasmées que la mondialisation culturelle est capable de susciter via la circulation densifiée des idées et des images.

Dans les cités, chaque groupe cherche ses marques en tant que collectif en projetant sur l'«Autre» des perceptions parfois caricaturales qui peuvent s'incarner dans les représentations spatiales comme l'illustre le cas du grand ensemble de SarcellesLochères. Si les Juifs y renvoient l'image stéréotypée de l'aisance financière, de la solidarité communautaire et de l'avarice ${ }^{13}$, les jeunes Antillais restent associés de manière toute aussi abusive au tryptique "artistes-sportifs-dealers». Dans ce vaste ensemble résidentiel multiethnique de 37000 habitants, ces perceptions investissent l'espace mental des habitants, le secteur plus aisé des Cholettes étant désigné comme le «quartier juif » tandis que le «Bas » de Lochères est considéré comme le territoire des Maghrébins (Sablons) et des Noirs (Valéry-Watteau). Quoique le risque communautariste ne soit pas absent, il convient cependant de ne pas survaloriser ces représentations que nuancent les pratiques spatiales. Dans les faits, nombre de commerces du quartier dits « ethniques » sont fréquentés par différents groupes et le marché forain des Lochères s'apparente à une véritable mosaïque.

Dans le contexte états-unien, le fait communautaire apparait nettement plus affirmé socialement et marqué géographiquement pour deux raisons: il s'appuie sur une reconnaissance institutionnelle dans des quartiers où la mixité culturelle est bien moins importante; l'appropriation mentale des espaces de résidence et de vie par les groupes ethnicisés se projète conséquemment sur des territoires beaucoup plus vastes et circonscrits dans la ville. Chez les Antillais de Miami, aujourd'hui majoritaires dans l'aire métropolitaine, la distance sociale et spatiale se construit sur fond de catégorisation ethnique et de hiérarchisation socio-économique. Cette distance se nourrit également de la méconnaissance de l'« Autre ». Le préjugé de couleur s'incarne dans des représentations réciproques territorialisées entre populations caribéennes. 
39 À travers leurs perceptions géographiques, les Antillais créolophones et anglophones mettent l'accent sur leur différenciation sociale et culturelle vis-à-vis des populations hispano-antillaises. La place limitée qu'occupent les secteurs latino-américains dans l'espace perçu des Haïtiens révèle la segmentation sociale et spatiale de cette ville où les populations noires et hispaniques des quartiers centraux tendent à vivre dans des quartiers séparés, à l'exception de certaines portions de Little Haiti. Les pratiques spatiales des Haïtiens ignorent généralement les quartiers latinos de Miami qui constituent pourtant les deux-tiers de la superficie de l'agglomération. Sauf exception, ils ne cotoyent régulièrement les Hispano-caribéens que dans le monde du travail et de manière ponctuelle. Ces rapports limités sont le fruit de l'absence d'un sentiment de cohésion sociale à l'échelle de la métropole liée à l'absence d'espaces de rencontre entre populations d'origines différentes et vivant dans des quartiers différents. Cette situation tend à favoriser les replis communautaires qui se traduisent dans les représentations spatiales par la méconnaissance de larges portions de l'espace urbain (Audebert, 2006a).

40 Les Antillais noirs non hispaniques assimilent les quartiers cubains à des zones étrangères dans lesquelles ils sont aisément identifiables, la cause n'étant plus leur accent, leur manière de marcher ou de s'habiller comme dans les quartiers noirs étatsuniens, mais la couleur de leur peau. Les secteurs noirs états-uniens quant à eux apparaissent comme clairement répulsifs pour les Antillais, du fait de l'image que les immigrants ont de la population noire autochtone (idem). Ils mettent en exergue quatre types de préoccupations: l'insécurité qui y règne; la perception de trop grandes différences en termes de modes de vie entre les Haïtiens et les Noirs autochtones; le sentiment de ne pas être chez soi dans ces quartiers; l'idée que ces lieux sont abandonnés par les pouvoirs publics. Dans le même temps, chaque communauté cherche à s'approprier de manière exclusive ce qu'elle considère comme son territoire comme l'illustre la dynamique des gangs de jeunes Antillais reproduisant celle des Noirs états-uniens. La création du gang haïtien Zoe Pound dans les années 1990 avait ainsi pour objectif l'élimination des bandes noires états-uniennes du territoire de Little Haiti qu'il considérait comme le sien.

\section{Les Antillais dans l'espace de représentation politique en France et aux États-Unis : vers une ethnicisation du rapport au territoire?}

41 Au-delà de la territorialisation résidentielle et cognitive, le rapport à l'espace des Antillais dévoile d'autres modes plus avancés de formalisation communautaire. La vie associative et les formes de représentation politique connaissent notamment de nouveaux développements dont les modalités divergent dans les deux pays. La prise de conscience des difficultés d'intégration nourrit un sentiment communautaire pouvant se traduire par une emprise croissante des catégories ethniques dans les techniques de mobilisation collective (Constant, 2000). La différence entre les cadres socioinstitutionnels français et états-unien réside dans la manière dont les solidarités s'exprimant sur une base communautaire ont été formalisées. L'émergence du fait communautaire sur la base de l'origine nationale - ou géographique pour les Domiens -, d'attributs culturels ou du phénotype a consacré le territoire comme un enjeu de l'ethnicisation plus ou moins marquée des rapports sociaux, que la 
participation des Caribéens à la vie politique locale permet de saisir. Si, comme on l'a analysé antérieurement, les réseaux et l'identité communautaires structurent l'espace, le territoire urbain peut aussi produire en retour de la relation et de l'identité ethnique de manière plus ou moins affirmée selon le contexte socio-institutionnel.

\section{L'émergence politique des Antillais aux États-Unis : des stratégies communautaristes s'incarnant dans le territoire}

Dans la continuité de l'affirmation des droits civiques des années 1960, la spécificité du système politique local états-unien réside dans l'accession au pouvoir à divers échelons de représentants communautaires liée à la constitution de territoires électoraux ethniques (redistricting). Cette territorialisation institutionnalise l'ethnicité en la confortant par la représentation politique. Il s'agit de faire correspondre les circonscriptions électorales à la géographie résidentielle des populations ethnicisées afin d'assurer une représentation équitable des minorités (tâche peu aisée sur le terrain et s'opérant au détriment des groupes les moins nombreux dans les zones ainsi redessinées). Or, la segmentation ethno-résidentielle des villes nord-américaines est à l'origine des fortes concentrations spatiales caribéennes remarquées précédemment dans certains quartiers. Cette distribution résidentielle rend directement compte de la genèse de vastes territoires électoraux antillais : Jamaïcains et Portoricains à New York, Cubains, Haïtiens et Jamaïcains en Floride.

Dès l'entre-deux-guerres, le tissu associatif portoricain de New York s'est structuré autour de la lutte contre les discriminations symbolisée par la liga puertorriqueña et prolongée dans les années 1960 par une intense activité syndicale. C'est suite à la lutte pour les droits civiques que la communauté portoricaine a enregistré ses premiers succès politiques. Ces derniers ont été symbolisés par l'élection d'Herman Badillo à l'échelon municipal new-yorkais dès 1965 puis au Congrès dès 1970. En 1992, une deuxième portoricaine a été élue au Congrès, alors que la communauté devenait très active au parlement de l'État de New York et au conseil municipal de New York City.

Confirmant le rôle stratégique de la concentration résidentielle des Antillais, le Bronx et Harlem se sont dès lors affirmés comme les épicentres de la vie politique portoricaine aux États-Unis. Il est vrai que les 320000 Portoricains du Bronx représentent près de $10 \%$ de la population portoricaine du pays (U.S. Census Bureau, 2001) et qu'ils bénéficient du soutien massif d'une population hispanique (dominicaine notamment) qui constitue près de la moitié de la population de ce comté. Le poids politique portoricain s'appuie plus fondamentalement sur une pratique ancienne des institutions locales et sur une citoyenneté états-unienne qui leur confère une large base électorale.

Plus récemment, les communautés antillaises non hispaniques ont aussi enregistré une émergence politique sans précédent dans le Nord-Est des États-Unis et en Floride. Le lobbying politique des Haïtiens était apparu dès les années 1970 en Floride pour répondre aux besoins considérables d'une population pauvre sans papiers. Une nébuleuse d'institutions communautaires à caractère culturel, social ou juridique s'était alors développée localement, avec pour objectif d'offrir aux migrants un environnement familier et protecteur et un écho auprès des autorités. Mais c'est l'accession massive à la citoyenneté états-unienne des Haïtiens dans les années 1990 qui leur a conféré une visibilité croissante - fait nouveau - sur le plan électoral. 

pays d'origine, les deux dernières décennies ont été marquées par un changement majeur de ce point de vue. La législation de 1986 favorable à l'accession à la résidence permanente a eu pour conséquence l'augmentation de l'effectif des individus devenant citoyens états-uniens. Cette tendance a en particulier été sensible à partir de $1996 \mathrm{du}$ fait de deux facteurs: la perte d'espoir d'un retour en Haïti et une législation migratoire supprimant certaines aides fédérales aux immigrés n'ayant pas la citoyenneté états-unienne. 149500 Haïtiens sont devenus citoyens états-uniens entre 1980 et 2001 , dont $61 \%$ entre 1996 et 2001 !

La concentration résidentielle des Haïtiens a accentué leur visibilité électorale. La Floride a en particulier concentré $33 \%$ de l'effectif total des nouveaux citoyens étatsuniens originaires d'Haïti entre 1989 et 2000 (figure 1). Cette évolution reflète le poids démographique croissant de la communauté haïtienne de Floride, mais également la volonté grandissante de ses membres d'exister politiquement dans la société d'accueil pour défendre leurs intérêts.

Figure 1 : Évolution des effectifs annuels d'Haïtiens ayant accédé à la citoyenneté états-unienne en Floride et dans les autres États des États-Unis entre 1989 et 2000

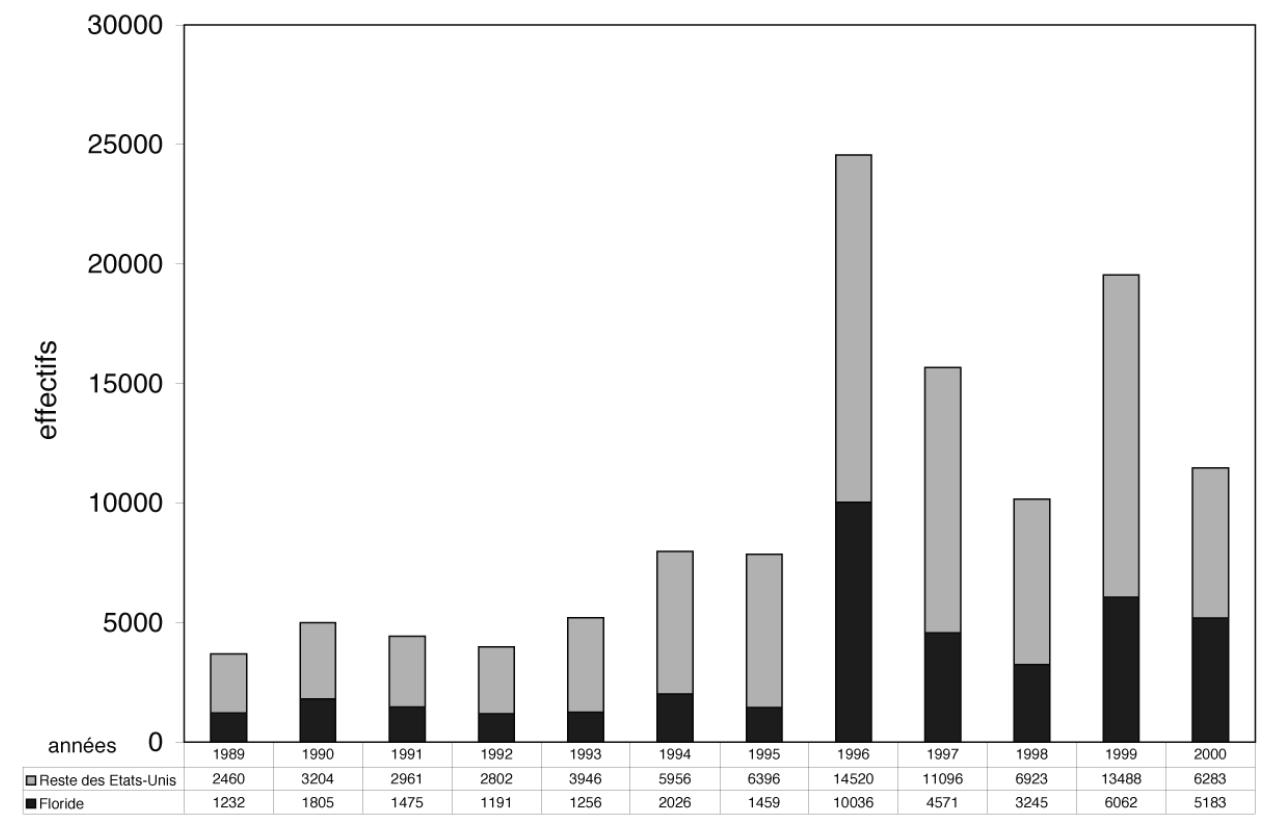

Note : 1989-1998 : individus de citoyenneté haïtienne ; 1999-2000 : individus nés en Haïti.

Source : U.S. Immigration and Naturalization Service Statistical Yearbooks [1990: table 55; 1991: table 51; 1992: table 47; 1993-1995: table 51; 1996-1999: table 50; 2000: table 49; 2001: table 52].

Ils comptent aujourd'hui deux élus au parlement de Floride et une dans le Massachussets. Le comté de Miami-Dade s'affirme comme le centre de leur ascension politique récente dans le pays, avec six élus dont quatre à l'échelon municipal. Le seul secteur nord-est de ce comté concentre $17 \%$ des originaires d'Haïti vivant dans le pays (U.S. Census Bureau, 2002) et $40 \%$ d'entre eux y ont acquis la citoyenneté étatsunienne. Des territoires électoraux comme ceux de North Miami sont constitués pour moitié de nouveaux électeurs haïtiens. On comprend dans ces conditions que ces espaces soient devenus les épicentres de l'ascension politique de ce groupe ethnique aux Etats-Unis. Dotés d'une forte conscience politique liée à leur marginalisation 
économique et sociale et à leur volonté de peser sur les décisions locales en tant que groupe ethnique, leur taux d'abstention moyen pour les élections locales est le plus faible de toutes les communautés : $15 \%$ seulement, alors qu'il est de $39 \%$ pour les Cubains, $75 \%$ pour les anglos et $79 \%$ pour les Noirs états-uniens ${ }^{14}$.

Signe de l'ethnicisation des rapports sociaux dans cette société, les rares solidarités intra-caribéennes ne parviennent pas à s'affranchir du carcan ethno-racial institutionnel. En effet, à une autre échelle que celle d'une mobilisation sur la base de l'origine nationale, elles se négocient sur la base de la catégorie raciale et de la langue parlée. D'un côté, les Haïtiens, les Jamaïcains et les autres Antillais anglophones élaborent des stratégies politiques communes de circonstance dans les circonscriptions électorales de Brooklyn (New York) et du comté de Broward (Floride) où ils cohabitent. De l'autre, les Cubains à Miami et les Portoricains et Dominicains à New York restent les noyaux durs de blocs politiques hispaniques autour desquels s'agrègent les autres populations hispano-caribéennes (Audebert, 2006b).

\section{Les Antillais dans l'espace public français : de la mobilisation communautaire à l'émergence de nouvelles identités}

Le contrat social républicain implique une invisibilité formelle du fait ethnocommunautaire dans la vie politique, non reconnu en tant que tel dans l'espace public. La vie électorale n'est donc pas a priori susceptible d'être investie par une éventuelle dimension ethnique comme aux États-Unis. Les Caribéens - franco-antillais ou haïtiens - malgré leur concentration résidentielle et leur vote plutôt à gauche n'interviennent donc pas en tant que communautés dans la vie politique locale française. Doit-on pour autant en conclure à leur absence du jeu politique en tant que collectif dans l'Hexagone?

51 Michel Giraud et Claude-Valentin Marie ont bien montré que la dimension politique de la vie sociale des Antillais s'est manifestée depuis les années 1980 plutôt dans la sphère associative, la seule à permettre une mobilisation communautaire que les syndicats et les partis politiques n'acceptent pas (Giraud, Marie, 1987). En réponse aux attentes déçues de migrants qui avaient idéalisé la «Mère patrie " mais dont la situation économique et sociale dans l'Hexagone est restée mitigée, un certain nombre de revendications spécifiques ont été présentées dans une dynamique progressive de mobilisation collective. Le cadre associatif favorable à l'épanouissement de l'identité culturelle s'est affirmé comme un "instrument de "l'institutionnalisation" d'une communauté en voie de consolidation " (idem). La vie associative antillaise s'est longtemps incarnée dans un foisonnement d'organisations culturelles pour maintenir le lien symbolique avec les sociétés d'origine et, si possible, transmettre l'héritage antillais aux générations suivantes. Les associations professionnelles de branches du secteur public où les Antillais étaient très présents (PTT, Assistance publique) ont constitué un pôle majeur de cette vie associative.

52 Cependant, dans un contexte où les populations dites minoritaires restent sousreprésentées dans l'arène politique, l'émergence de figures charismatiques développant un discours à forte empreinte identitaire et convoquant une histoire caribéenne douloureuse a pu - ponctuellement mais de manière inédite - mobiliser une partie de l'électorat antillo-guyanais sur une base proprement communautaire. Quoique non investi d'une dimension ethnique institutionnelle, le territoire électoral a alors joué 
une fonction inattendue dans la visibilité politique de la population antillaise. Lors du premier tour des élections présidentielles de 2002, la candidate guyanaise Christiane Taubira a réalisé ses meilleurs scores hexagonaux dans les circonscriptions enregistrant une forte présence de l'électorat antillais. Dans ces secteurs, la candidate y a systématiquement réalisé des résultats trois fois à quatre fois supérieurs à son score national (2,32\%), dessinant une véritable cartographie de la présence antillo-guyanaise dans l'Hexagone avec notamment des résultats entre $8 \%$ et $10 \%$ à Bobigny, Créteil, Sarcelles/Garges et Saint-Denis/Stains ${ }^{15}$.

Néanmoins, à la différence des États-Unis, cette mobilisation électorale s'effectuant en partie sur une base communautaire ne s'est jamais traduite par une identification massive, systématique et durable à un parti ou une figure politique et encore moins par une ethnicisation de la population en question par les institutions de la République. Ici, la territorialisation du vote antillo-guyanais apparaît davantage comme un révélateur de préoccupations sociales et identitaires particulières jusqu'ici peu prises en compte et non pas comme le support institutionnel de stratégies communautaristes. Il reste qu'au cours des années 1990 et 2000, les difficultés croissantes des Antillais et des générations suivantes à s'affirmer individuellement dans la sphère socio-économique les a amenés à renforcer leur mobilisation collective sur le mode de la revendication identitaire, à l'instar de leurs aînés vingt ans auparavant mais selon des méthodes différentes. L'année 2005 a constitué un tournant de ce point de vue, avec l'importance croissante donnée à la question de l'héritage de la colonisation, à la mémoire de la traite et de l'esclavage et au débat toujours sous-jacent de la discrimination positive ravivé sur fond de tensions dans les banlieues. Cette question s'est inscrite dans le prolongement de la loi Taubira reconnaissant en 1998 l'esclavage comme crime contre l'humanité et donnant plus d'importance à ce fait historique dans les programmes scolaires.

La formalisation croissante de la vie associative qui en a résulté traduit la diversité des stratégies communautaires et des projections identitaires parmi les originaires des Antilles, que deux associations fédératrices aux desseins divergents symbolisent assez bien : le Collectifdom (Collectif des Antillais, Guyanais, Réunionnais et Mahorais) et le CRAN (Conseil représentatif des associations noires). La première se fonde sur la citoyenneté et l'origine géographique, puisqu'elle milite pour l'égalité des droits, la représentation de la France dans toute sa diversité et lutte contre les discriminations concernant les Français originaires d'outre-mer dans les domaines de l'emploi et du logement notamment. La seconde se revendique également comme une organisation citoyenne, au sens où sa raison d'être réside dans la lutte contre les discriminations «raciales». Leurs orientations distinctes révèlent de manière plus générale la multiplicité des stratégies identitaires des Antillais en France, en réponse aux enjeux posés par la discrimination et la volonté de reconnaissance de leurs apports historiques et culturels au creuset français.

55 Certains individus privilégient la relation concrète ou symbolique avec les espaces d'origine et développent l'essentiel de leurs réseaux sociaux dans un cadre communautaire. D'autres projètent leur destin dans l'Hexagone et lorsqu'ils valorisent un fonctionnement intra-communautaire (ce qui est loin d'être toujours le cas), mettent en exergue une identité des Antillais - ou des domiens - de France. Enfin, une troisième stratégie met en exergue une communauté de destin fondée sur la couleur de la peau, plus que sur des considérations culturelles ou une solidarité liée à l'origine géographique. Elle apparaît largement favorable aux échanges et solidarités entre 
Antillais et Africains et à l'énonciation de revendications communes. Mais au-delà des clivages précités, la réalité n'est pas aussi segmentée et il n'est pas rare qu'un même individu mette en exergue simultanément ces trois types de stratégies identitaires.

Outre leur " antillanité de France ", les générations nées dans l'Hexagone semblent de plus en plus privilégier une identité de banlieue sur la base de revendications sociales transcendant l'ethnicité et dont l'action a pour supports les quartiers populaires et pour objectif leur meilleure représentation politique. C'est probablement l'un des principaux enseignements de la mobilisation électorale à l'initiative entre autres du collectif Devoirs de mémoires suite aux émeutes de $2005^{16}$. Cette formalisation associative traduit aussi le besoin de politisation d'une population "minorisée " à laquelle les syndicats et partis politiques traditionnels n'ont offert que peu de champ pour exprimer sa citoyenneté. Il s'agit là encore d'une différence remarquable avec les États-Unis, où les institutions ont autorisé et encouragé la représentation politique des groupes ethnicisés jusque dans les plus hautes sphères du pouvoir.

\section{Conclusion}

57 L'implantation durable des Antillais en France et aux États-Unis implique des projections à long terme dans la société métropolitaine et des préoccupations et défis à relever différents de ceux des Antillais évoluant dans les sociétés d'origine. Les principaux enjeux les concernant, liés à l'appartenance citoyenne, renvoient aux problématiques de la participation et de la reconnaissance de leur héritage et de leurs apports à la construction des sociétés d'installation. Ces enjeux résident dans un équilibre entre l'insertion dans la structure socio-économique métropolitaine et la reformulation réussie d'une identité propre en cohérence avec leur expérience migratoire et post-migratoire.

La mise en parallèle des expériences des Portoricains, Antillais français et Haïtiens montre que si la possession formelle de la citoyenneté facilite incontestablement les conditions d'entrée et d'insertion sur le territoire métropolitain, elle ne constitue pas un rempart contre l'inégalité d'accession aux droits sociaux et à la participation politique. Elle ne garantit pas systématiquement pour tous, les mêmes conditions d'appartenance et de participation à la vie de la cité. Cela apparaît en particulier dans le contexte d'une représentation à géométrie variable de la communauté nationale.

L'expérience comparée de ces populations permet aussi de remettre en perspective les systèmes socio-institutionnels français et états-unien, en démystifiant la dimension paradigmatique et parfois idéologique de visions les érigeant en sociétés diamétralement opposées. Au-delà de traitements spécifiques de la différence culturelle, et du fait de leur héritage démocratique et colonial, la France et les ÉtatsUnis partagent à la fois un idéal universel fondant leur identité nationale et une réalité pluriculturelle amenant à repenser en permanence leur conception de la citoyenneté et leur rapport à l'altérité. La question de l'insertion (relégation?) des populations ethnicisées dans les structures sociales et économiques et celle plus ou moins adroitement formulée de la reconnaissance (reformulation?) identitaire renouvellent les enjeux de la citoyenneté auxquels les deux sociétés sont également confrontées, en dépit de conditions historiques et institutionnelles distinctes de production de l'altérité. 
60 l'identité et de la citoyenneté s'incarnent dans le territoire. Les enjeux sociaux d'un investissement identitaire des espaces locaux plus ou moins formalisé tiennent à l'invention permanente de nouvelles formes d'expressions du lien social et de l'appartenance à la nation. L'identité de territoire révèle alors des conceptions divergentes du "vivre ensemble» dans les deux pays, puisqu'elle est formulée sur la base de l'ethnicité aux États-Unis alors qu'elle l'est plutôt sur celle d'une revendication sociale en France qui transcende la qualification ethnique - sans pour autant toutefois l'ignorer.

61 Enfin, les modalités de territorialisation des Caribéens dans les grandes métropoles des deux côtés de l'Atlantique portent à nuancer la représentation de ces quartiers, certes souvent peu favorisés, comme des espaces de marginalisation et d'enfermement. L'altérité porteuse de ségrégation et d'assignation identitaire a des effets ambivalents, contradictoires qui ne se résument pas à la simple relégation des populations concernées. Loin de n'être que stigmatisante, la visibilité des populations qu'elle conforte peut aussi favoriser dans certains contextes une meilleure représentation politique.

\section{BIBLIOGRAPHIE}

ANDERSON Benedict (1991) Imagined communities: reflections on the origin and spread of nationalism, New York \& London, Verso, 224 p (première éd. 1983).

AUDEBERT Cédric (2006a) L'insertion socio-spatiale des Haïtiens à Miami, Paris, coll. Populations, L'Harmattan, $301 \mathrm{p}$.

AUDEBERT Cédric (2006b) Les communautés antillaises aux États-Unis : entre métropolisation et logiques réticulaires transnationales, Espace Populations Sociétés, 2006 (1), pp. 137-149.

AUDEBERT Cédric (2002) Le fait religieux dans l'insertion et l'organisation spatiale de la communauté haïtienne de Miami, Géographie et Cultures, 43, pp. 107-127.

BASCH Linda, GLICK SCHILLER Nina, SZANTON BLANC Cristina (Dir.) (1994) Nations unbound: transnational projects, postcolonial predicaments and deterritorialized nation-states, New York \& Londres, Routledge, $344 \mathrm{p}$.

BODY-GENDROT Sophie (1988) Les immigrants dans la vie politique en France et aux États-Unis, Revue européenne des migrations internationales, 4 (3), pp. 7-22.

BOURDIEU Pierre (1993) Effets de lieu, in Pierre Bourdieu (Dir.), La misère du monde, Paris, Seuil, pp. 159-167.

COHEN Jim (2002) Diaspora et incorporation : présences publiques des Caribéens aux États-Unis, Hommes et Migrations, 1237.

CONDON Stéphanie (1994) L'accès au logement : filières et blocages. Le cas des Antillais en France et en Grande-Bretagne, Population, 49 (2), pp. 522-530.

Revue européenne des migrations internationales, vol. 24 - $n^{\circ} 1$ | 2008 
CONSTANT Fred (2000) La citoyenneté, Paris : Montchrestien.

CONSTANT Fred (1987) La politique française de l'immigration antillaise de 1946 à 1987, Revue Européenne des Migrations Internationales, 3 (3), pp. 9-30.

DESPLAN Fabrice (2005) Le religieux minoritaire comme outil de reconstruction identitaire. Le cas des Antillais français adventistes en France, ethnographies.org, $\mathrm{n}^{\circ} 8$ [en ligne], 33 p. http:// www.ethnographiques.org/2005/desplan.html (consulté le 30 juin 2007)

DOMENACH Hervé, PICOUËT Michel (1992) La dimension migratoire des Antilles, Paris, Economica, $254 \mathrm{p}$.

GIRAUD Michel (2002) Racisme colonial, réaction identitaire et égalité citoyenne : les leçons des expériences migratoires antillaises et guyanaises, Hommes et Migrations, 1237, pp. 40-53.

GIRAUD Michel, MARIE Claude-Valentin (1987) Insertion et gestion socio-politique de l'identité culturelle : le cas des Antillais en France, Revue européenne des migrations internationales, 3 (3), pp. 31-48.

GREEN Nancy L. (2002) Repenser les migrations, Paris, PUF, 139 p.

GROSFOGUEL Ramon (2002) La problématique intégration des Portoricains aux États-Unis, Hommes et Migrations, 1237, pp. 91-100.

GROSFOGUEL Ramon (1999) Introduction: "cultural racism" and colonial Caribbean migrants in core zones of the capitalist world-economy, Review, 22, pp. 409-434.

INSEE (Institut National de la Statistique et des Études Économiques) (1999) Recensement général de la population 1999, CD-ROM.

LACORNE Denis (1997) La crise de l'identité américaine : du melting-pot au multiculturalisme, Paris, Fayard, 394 p.

MARIE Claude-Valentin (2002) Les Antillais en France : une nouvelle donne, Hommes et Migrations, 1237, pp. 26-39.

MASSEY Douglas, DENTON Nancy (1993) American apartheid: Segregation and the making of the underclass, Cambridge \& Londres, Harvard University Press, 292 p.

NOIRIEL Gérard (1988) Le creuset français : histoire de l'immigration (XIX $-\mathrm{XX}^{\mathrm{e}}$ siècle), Paris, Le Seuil, $437 \mathrm{p}$.

SCHNAPPER Dominique (1994) La communauté des citoyens. Sur l'idée moderne de nation, Paris, Gallimard, 202 p.

STEPICK Alex (1998) Pride against prejudice : Haitians in the United States, Boston, Allyn and Bacon, $134 \mathrm{p}$.

U.S. CENSUS BUREAU (2002) Census of population and housing 2000, Summary file 3.

U.S. CENSUS BUREAU (2001) Census of population and housing 2000, Summary file 1.

U.S. CENSUS BUREAU (1999) Current Population Survey (CPS).

U.S. DEPARTMENT OF HOUSING AND URBAN DEVELOPMENT (2000) The state of cities: 2000, Washington, U.S. Government, 107 p.

WILSON W.J. (1987) When works disappears: the world of the new urban poor, Random House, 352 p. 


\section{NOTES}

1. Nous définissons comme Antillais l'ensemble des individus originaires des territoires insulaires bordant la Mer des Antilles. Les Haïtiens et les Portoricains seront donc considérés comme des Antillais au même titre que les Antillais français. Par extension, nous parlerons d'AntilloGuyanais lorsqu'il s'agira d'inclure l'expérience des Guyanais dans le contexte français.

2. Pour une vision globale de la question, on consultera avec profit le numéro 1237 d'Hommes \& Migrations consacré aux migrations caribéennes paru en 2002.

3. Les originaires des Antilles françaises seraient 337000 dans l'Hexagone (Marie, 2002), dont 211 800 migrants (INSEE, 1999). Les ressortissants d'Haïti y seraient 24 900, un chiffre inférieur de moitié aux estimations des associations de migrants et des institutions religieuses. OutreAtlantique, les natifs d'Haïti représenteraient l'équivalent de $79 \%$ de l'effectif des originaires d'Haïti (531 400), à mettre en parallèle avec les natifs de Porto-Rico constituant l'équivalent de $42 \%$ de l'effectif de la communauté portoricaine de 3406000 individus (U.S. Census Bureau, 2002 ; 2001). Cette différence entre les deux communautés est liée à la présence plus ancienne des Portoricains dans la société d'accueil par rapport à celle des Haïtiens. Le poids relatif des générations suivantes invite à porter un regard critique sur la notion même d'intégration et à affiner notre lecture des dynamiques d'interaction qui la constituent.

4. La référence à l'ethnicité ne préjuge ici d'aucun essentialisme ni d'aucune limite figée ou naturelle censée déterminer l'appartenance à tel ou tel groupe. Privilégiant le point de vue des acteurs, elle se fonde plutôt sur la croyance partagée en une origine commune réelle ou supposée, souple et évolutive par définition.

5. Dans les aires métropolitaines états-uniennes, les emplois de services ont progressé dix fois plus vite que les emplois manufacturiers ( $21 \%$ contre 2,4\%) au cours des années 1990. Mais surtout, l'emploi manufacturier traditionnel des inner cities a enregistré une contraction de 5,4 \% (U.S. Department of Housing and Urban Development, 2000).

6. La mention "DT" apposée par les recruteurs de certaines entreprises sur les dossiers des originaires des DOM-TOM pour les écarter illustre les discriminations à l'embauche dont ils peuvent faire l'objet.

7. Bureau des migrations intéressant les travailleurs des Départements d'outre-mer.

8. L'indice de ségrégation des Antillais noirs vis-à-vis des Blancs non hispaniques était de 0,72 aux États-Unis en 2000 (U.S. Census, 2000), ce qui signifiait que $72 \%$ des Antillais devraient changer de quartier pour que leur distribution spatiale soit similaire à celle des Blancs dans tous les quartiers de l'aire métropolitaine.

9. Ne pouvant traiter de l'ensemble des aspects de la vie communautaire des populations antillaises en migration, l'analyse se limitera ici à la dimension religieuse. Pour une analyse détaillée de la structuration sociale et spatiale des quartiers antillais par l'entreprenariat ethnique aux États-Unis, voir Audebert (2006a).

10. Les secteurs de Cergy-Pontoise, Saint-Denis/Stains/Epinay/La Courneuve, Aulnay/ Sevran/ Villepinte, Sarcelles/Garges/Villiers-le-Bel, et des $18^{\text {ème }}, 19^{\text {ème }}$ et $20^{\text {ème }}$ arrondissements de Paris sont des espaces de forte cohabitation entre Antillais français, Haïtiens et Subsahariens. Sarcelles, souvent présentée comme la principale concentration caribéenne de l'Hexagone, compte à peine $10 \%$ d'Antillais. Ces derniers y cohabitent avec plusieurs milliers de Juifs sépharades, de Maghrébins, de Chaldéens et de Subsahariens.

11. Les termes du verlan «Babtou» (Toubab, Blanc), «Renoi » (Noir), «Rebeu » (Beur, Arabe) et «Feuj » (Juif) sont couramment employés dans les cités pour désigner les populations précitées.

12. La population noire apparaît ainsi la seule dans le contexte français à être désignée par un vocable anglo-saxon.

13. L'expression «Fais pas ton Feuj » est couramment utilisée dans les cités pour dire « Ne soit pas radin ». 
14. Source : Miami-Dade Department of Elections.

15. Source: Ministère de l'Intérieur. Quoique la correspondance entre le vote Taubira et les secteurs de concentration résidentielle antillo-guyanaise apparaisse remarquable, seule une analyse statistique régressive permettrait de montrer de manière irréfutable l'existence d'un vote communautaire lors de ces élections. Cela constituerait en soi l'objet d'un autre article.

16. Les originaires des Antilles constituent près du tiers des personnalités ayant parrainé cette initiative (Devoir de réagir), dont notamment Joey Starr, Lilian Thuram, Admiral T, Jacques Martial, Brother Jimmy ou Daddy Nuttea.

\section{RÉSUMÉS}

Une comparaison de l'expérience sociale des populations d'origine antillaise en France et aux États-Unis révèle une intégration ambivalente dans les communautés nationales, que traduit leur «minorisation». La formalisation spatiale de l'altérité se décline selon des modalités dissemblables de territorialisation dans les deux pays. Les concentrations résidentielles, la territorialisation communautaire et les représentations spatiales marquent ainsi des logiques sociétales distinctes propres à chaque contexte. De même, l'analyse de la visibilité différenciée des Antillais dans l'espace de représentation politique en fonction du contexte national montre que si l'on observe dans les deux cas des processus d'ethnicisation des territoires, l'institutionnalisation du rapport à l'espace aux États-Unis a pour conséquence une émergence politique des Antillais que l'on n'observe pas en France.

The incorporation of Caribbeans in France and the United States: socio-institutional contexts and territorialization processes. A comparative perspective on the social experience of populations of Caribbean descent in France and the U.S reveals their ambivalent incorporation as minorities in the national communities. The construction of otherness is formalized differently in both countries. Residential concentration, the territorialisation of ethnic communities and spatial perceptions show distinct societal evolutions which are specific to each national context. Likewise, the analysis of the differentiated visibility of Caribbeans in the political arena depending on the national context shows that despite the ethnicisation of neighborhoods, the institutionalization of the relation to the local territory in the U.S. results in a Caribbean political emergence which is not observed in France.

La integración de los Antilleses en Francia y a los Estados Unidos: contextos socioinstitucionales y proceso de territorialización. Una comparación de la experiencia social de las poblaciones de origen antillés en Francia y a los Estados Unidos revela una integración ambivalente en las comunidades nacionales. La formalización espacial de la alteridad se declina según modalidades diferentes de territorialización en los dos países. Las concentraciones residenciales, la territorialidad comunitaria y las representaciones espaciales señalan así lógicas empresariales distintas consustanciales a cada contexto. Del mismo modo, el análisis de la visibilidad diferenciada de los Antilleses en el espacio de representación político en función del contexto nacional pone de manifiesto que si se observa en los dos casos unos procesos de etnicización de los territorios, la institucionalización de la relacion al espacio en los Estados Unidos tiene por consecuencia una emergencia política de los Antilleses que no se observa en Francia. 
INDEX

Index géographique : États-Unis, France

Mots-clés : espace, ethnicisation, intégration sociale, représentations, territorialisation, Antillais

\section{AUTEUR}

\section{CÉDRIC AUDEBERT}

Chargé de recherche à Migrinter (CNRS, Université de Poitiers) 99, av. du Recteur Pineau, 86000 Poitiers, cedric.audebert@univ-poitiers.fr 